\title{
7 Patientenbeteiligung bei medizinischen Entscheidungen als Qualitätsmerkmal der Versorgung am Beispiel der ambulanten Depressionsbehandlung
}

Andreas Loh und Sabine Beck

\subsection{Mit welchen Argumenten ist Patientenbeteiligung zu rechtfertigen?}

Die Beteiligung von Patienten bei der medizinischen Entscheidungsfindung nach dem Konzept des Shared Decision-Making (Charles et al. 1997 und 1999) wird nicht nur deshalb umgesetzt, um Patientenbedürfnissen gerecht zu werden und eine patientenorientierte Medizin zu ermöglichen, sondern entwickelt sich zunehmend als ein fester Bestandteil der Qualitätsbewertung medizinischer Versorgung. Viele Patienten, Ärzte, Pflegende und Vertreter der Forschung, der Krankenkassen und der Gesundheitspolitik fordern oder unterstützen diesen Ansatz, weil hiermit eine ganze Reihe von wünschenswerten Effekten verbunden ist, was es letztlich auch rechtfertigt, Patientenbeteiligung als Qualitätsmerkmal zu verstehen.

Neben dem steigenden Bedarf der Patienten an Information und Beteiligung ist es auch aus rechtlichen Gründen gefordert, Patienten bei der medizinischen Entscheidungsfindung zu beteiligen. In einer Informationsschrift der Bundesministerien für Gesundheit und Justiz wird verpflichtend gefordert, sofern mehrere medizinische Behandlungen oder Behandlungsmethoden in Betracht kommen, dass über die Wahrscheinlichkeit von Risiken im Verhältnis zu den Heilungschancen der zur Verfügung stehenden Behand- 
lungsmöglichkeiten so aufzuklären ist, dass Patienten in die Lage versetzt werden beurteilen zu können, was die jeweilige Behandlung konkret für sie bedeuten kann.

Ein bedeutendes Argument für die Berücksichtigung der Patientenbeteiligung als Qualitätsmerkmal sind aber die empirischen Ergebnisse einer verbesserten Behandlungsbereitschaft und in einigen Studien eines größeren Behandlungserfolges. Bewertet man die Qualität einer Versorgungsleistung nicht in dem Sinn, dass das Versorgungsangebot, beispielsweise die Information über die zu erwartenden positiven und unerwünschten Ereignisse und die Einnahmemodalitäten einer medikamentösen Therapie, auf qualitativ hochwertige Art und Weise an den Patienten vermittelt wird, sondern auch dadurch, wie dieses Versorgungsangebot beim Patienten ankommt, dann ergibt sich zwangsläufig, dass auch Maßnahmen zur Verbesserung des Verständnisses einer Behandlung und zur Optimierung der Behandlungsbereitschaft von Patienten als Versorgungs-Qualität verstanden werden müssen. Patientenbeteiligung bei medizinischen Entscheidungen fördert Behandlungsbereitschaft und Umsetzung der gewählten Behandlung (Adhärenz) und begünstigt auf diesem Wege den Behandlungserfolg. In Überblicksarbeiten konnte belegt werden (O'Connor et al. 2002; Frosch et al. 1999; Scheibler et al. 2003 und Loh et al. 2007), dass die Partizipative Entscheidungsfindung zu höherem Wissen über Behandlungsmöglichkeiten, zu realistischeren Erwartungen über den Verlauf, zu verringerten Entscheidungskonflikten, zur Verbesserung der Qualität der Arzt-Patienten-Interaktion, zu beständigerer Umsetzung der gewählten Behandlung (Adhärenz), zu einer veränderten Therapiewahl, zu höherer Patientenzufriedenheit und sehr wahrscheinlich auch zu stärkeren Behandlungseffekten führt.

\subsection{Hindernisse und Erfolgsfaktoren für die Umsetzung}

Bei der konkreten Umsetzung des Ansatzes werden nachfolgend einige Voraussetzungen angesprochen, die bei der Umsetzung der Patientenbeteiligung zum Beispiel in der ambulanten Behandlung der Depression gegeben sein sollten.

Zunächst sind die unterschiedlichen Voraussetzungen bei Ärzten und Patienten sowie strukturelle Bedingungen des Gesundheitssystems bei der Umsetzung zu bedenken. Erfahrungsgemäß werden von Ärzten Beeinträchtigungen im strukturellen Sinne häufig genannt, wenn der Ansatz der Patientenbeteiligung bei medizinischen Entscheidungen thematisiert wird. Ärzte befürchten, dass der Ansatz angesichts des hohen Zeitdrucks in der alltäglichen Praxis nicht durchführbar ist. Demgegenüber hat sich beispielsweise in der ambulanten Depressionsbehandlung gezeigt, dass nicht mehr Konsultationszeit aufgewendet werden muss, sofern zwei Voraussetzungen gegeben sind (Loh et al. 2007): Erstens müssen die Ärzte im Konzept der Partizipativen Entscheidungsfindung eine Fortbildung erhalten haben, die sie dazu befähigt, 
7 Patientenbeteiligung bei medizinischen Entscheidungen als Qualitätsmerkmal der Versorgung am Beispiel der ambulanten Depressionsbehandlung

die Handlungsschritte des Ansatzes in der Arzt-Patienten-Kommunikation erfolgreich umzusetzen. Zweitens müssen spezifisch gestaltete Materialien vorliegen, mit denen die Patienteninformation unterstützt und der eigentliche Entscheidungsprozess in der Konsultation wirksam vorbereitet werden kann. Sowohl entsprechende Materialien oder Verlinkungen auf qualitätsgeprüfte Patienteninformationen als auch Angaben zu Fortbildungsmöglichkeiten sind auf der Internetseite www.patient-als-partner.de einzusehen.

Im konkreten Behandlungsprozess sollten zunächst der individuelle Informationsbedarf des Patienten und dessen Interesse, an der Entscheidungsfindung beteiligt zu sein, exploriert werden. Bezogen auf die Depressionsbehandlung befürchten viele Ärzte, dass die Patienten insbesondere durch die kognitiven Beeinträchtigungen und den verminderten Antrieb durch die Grunderkrankung weder willens noch in der Lage sind, Informationen aufzunehmen oder sich an der Entscheidungsfindung zu beteiligen. Diese Befürchtung erweist sich bei Betrachtung der Studienlage als unbegründet: Studienergebnisse zum Informationsbedarf und zum Interesse an einer Beteiligung bei der Entscheidungsfindung zeigen, dass der Informationsbedarf der depressiven Patienten sehr hoch ist und das Interesse an der Beteiligung bei Entscheidungen insgesamt etwas geringer, aber immer noch in einem beträchtlichen Ausmaß vorhanden ist und mit der Zunahmen des Schweregrades der Depression nicht abnehmen (Loh et al. 2004). Nun könnte man aber annehmen, dass dieser Bedarf von depressiv erkrankten Patienten wesentlich geringer ist als der Bedarf bei Patienten mit anderen und insbesondere körperlichen Erkrankungen. Jedoch zeigen auch hier Studienergebnisse, dass sich diesbezüglich depressive Patienten nicht von Patienten mit Bluthochdruck oder Brustkrebs unterscheiden (Hamann 2007). Insgesamt zeigen Studienergebnisse, dass Ärzte das Interesse der Patienten an Information und an Beteiligung bei der Entscheidungsfindung deutlich unterschätzen. Dass die Implementierung noch unzureichend ist, wurde beispielsweise durch die Arbeit von Braddock und Kollegen (1999) belegt, die bei 1057 auf Tonband protokollierten ärztlichen Beratungen feststellten, dass medizinische Basisentscheidungen nur in $0,5 \%$ der Fälle, Entscheidungen von mittlerer Komplexität nur in 4,6\% und komplexe Entscheidungen nur in 15,2\% der Fälle so besprochen wurden, dass eine informierte (Partizipative) Entscheidung mit Beteiligung des Patienten beobachtet werden konnte.

\subsection{Mit welchen Maßnahmen kann man Patientenbeteiligung implementieren?}

Zum Transfer der Partizipativen Entscheidungsfindung in die Versorgung werden unterschiedliche Interventionen diskutiert (Härter et al. 2004). An erster Stelle steht hierbei die Entwicklung und Bereitstellung von Entscheidungshilfen (Decision Aids) in Form von Broschüren, audiovisuellen Materialien oder zunehmend in Form von mehr oder weniger interaktiven webbasierten 
Informationsportalen. Mit diesen Materialien wird das Ziel verfolgt, dass Patienten sowohl über die Erkrankung und die verfügbaren Behandlungsalternativen informiert werden, als auch einen von den Materialien angeregten und unterstützen Prozess der Werteklärung durchlaufen können. Der Einsatz von Entscheidungshilfen führt zwar nicht unbedingt zur Patientenbeteiligung im Konzept der partizipativen Entscheidungsfindung, denn Decision Aids sind nicht vom Prinzip her mit der ärztlichen Konsultation verknüpft (Loh et al. 2007, DÄB) und könnten letztlich auch eine rein autonome Entscheidung des Patienten ohne Mitwirkung eines Arztes begünstigen. Dennoch wird in aller Regel durch die Verfügbarkeit dieses Materials die Patientenbeteiligung bei medizinischen Entscheidungen gefördert. Durch die vergleichsweise große Bedeutung der Information für die Entscheidungsfindung ist darauf zu achten, dass die Materialien von hoher Qualität und unbeeinflusst durch ökonomische Interessen sind. Hierzu wurden in der letzten Zeit Qualitätsstandards zur Entwicklung und zum Inhalt von Decision Aids vorgelegt (Elwyn et al. 2006). Im deutschen Sprachraum wurden hierzu in den letzten Jahren sinnvolle Entwicklungen vorgenommen und Materialien bereitgestellt, beispielsweise im Rahmen eines Patientenservices der Bundesärztekammer und der Kassenärztlichen Bundesvereinigung (www.patienten-information.de) oder auf den Internetseiten der Patientenleitlinien der Universität Witten/Herdecke (www.patientenleitlinien.de).

Der zweite Ansatzpunkt zur Implementierung der partizipativen Entscheidungsfindung besteht in Fortbildungsmaßnahmen für Ärzte oder Patienten. Dabei hat sich in Deutschland gezeigt, dass wesentlich mehr Interventionen zum Aufbau von ärztlichen Kompetenzen als von Patientenkompetenzen hinsichtlich der partizipativen Entscheidungsfindung durchgeführt worden sind (Härter et al. 2005). In einer Arbeitsgruppe am Universitätsklinikum Freiburg wurde eine mehrstufige Intervention zur Implementierung in der hausärztlichen Depressionsbehandlung durchgeführt, bei der ein spezifisches Fortbildungsprogramm mit der Bereitstellung von Patienteninformationsmaterialien und der Entwicklung von Entscheidungstafeln (sog. „decision boards“) verknüpft wurde (Loh et al. 2006 Bundesgesundheitsblatt). Es gibt bisher kein im wissenschaftlichen Konsens weithin akzeptiertes Curriculum zur Fortbildung in partizipativer Entscheidungsfindung. Einzelne Fortbildungsveranstaltungen werden derzeit meist im Rahmen von Interventionen in Forschungsprojekten umgesetzt. Auf der Internetseite www.patient-als-partner. de werden einzelne und regionale Fortbildungsveranstaltungen ausgeschrieben oder angekündigt. Fundierte Fortbildungsmaßnahmen zeichnen sich dadurch aus, dass szenische Gestaltungen beziehungsweise Rollenspielverfahren oder Simulationspatienten eingesetzt werden. Dabei sollte nicht nur exemplarisch „vorgespielt“ werden, sondern in aufeinander aufbauenden Schritten sind möglichst viele einzelne Handlungsschritte in einer beispielhaften Arzt-Patienten-Kommunikation von möglichst allen Teilnehmern der Veranstaltung darzustellen. 


\subsection{Wie wird Patientenbeteiligung in der Fortbildung und in der Praxis umgesetzt?}

Als Ausgangspunkt für die Umsetzung sollte zur Qualitätssicherung in der Arzt-Patienten-Kommunikation und bezüglich der hausärztlichen Depressionsbehandlung auf Leitlinien zurückgegriffen werden. Hier empfehlen sich Leitlinien zur hausärztlichen Gesprächsführung, in denen die Partizipative Entscheidungsfindung explizit berücksichtigt wird (Leitliniengruppe Hessen 2008) und Leitlinien zum hausärztlichen Vorgehen bei der Diagnose und Behandlung depressiver Erkrankungen (Härter et al. 2003).

Das konkrete Vorgehen im ärztlichen Gespräch orientiert sich an Handlungsschritten, mit denen keinesfalls ein standardisierter oder formaler Ablauf einer Konsultation begründet werden soll, sondern eine handlungsleitende Orientierung gegeben werden soll, die sich insbesondere für die Vermittlung des Ansatzes in der ärztlichen Aus- und Fortbildung als hilfreich erwiesen hat. Die Rollen von Arzt und Patient werden durch das explizit ausgesprochene Angebot der Zusammenarbeit beim Treffen der Behandlungsentscheidung bestimmt. Beide, Arzt und Patient, sind prinzipiell gleichberechtigt. Diese Gleichberechtigung oder Gleichwertigkeit bezieht sich auch auf die unterschiedlichen Behandlungsmöglichkeiten, sofern für diese evidenzbasierte Belege aus klinischen Studien vorliegen. Der Arzt vermittelt, dass es mehr als eine Möglichkeit zur Behandlung der Beschwerden gibt und dass jede davon unterschiedliche Chancen und Risiken hat.

In der Konsultation werden dann die jeweiligen Vor- und Nachteile der Behandlungsmöglichkeiten erörtert, auf dem Hintergrund der Lebenssituation des Patienten abgewogen und eine gemeinsame Entscheidung wird getroffen. Im weiteren Verlauf der Konsultation exploriert der Arzt die Sicht des Patienten im Hinblick auf Erwartungen oder Befürchtungen zu den Behandlungsoptionen. Danach erfolgt ein Abwägen der Optionen und ein Plan zur Umsetzung der gewählten Behandlung wird gemeinsam vereinbart.

Während des Erläuterns der Behandlungsmöglichkeiten erweist es sich als sinnvoll, auf Schaubilder zurückgreifen zu können, um dem Patienten im Gespräch das Vorhandensein von Wahlmöglichkeiten und die Vor- und Nachteile der zur Verfügung stehenden Möglichkeiten verdeutlichen zu können (s. Abb. 15). Bei den Angaben zu den Behandlungsmöglichkeiten sind je nach Schweregrad Antidepressiva und Psychotherapie sowie deren Kombination als Behandlungsoptionen in der hausärztlichen Depressionsbehandlung zu nennen.

Es ist erforderlich die wissenschaftlichen Erkenntnisse so darzustellen, dass der Patient ihre Bedeutung für seinen Lebensalltag ableiten und erwägen kann. Dazu ist im ärztlichen Gespräch ein Perspektivenwechsel erforderlich. Die Detailinformationen beispielsweise über Ansprechraten, Wirkungs- oder Nebenwirkungsprofile sind so zu besprechen, dass der Patient diese im Hinblick auf seine eigenen Entscheidungskriterien und bezüglich seiner eigenen 


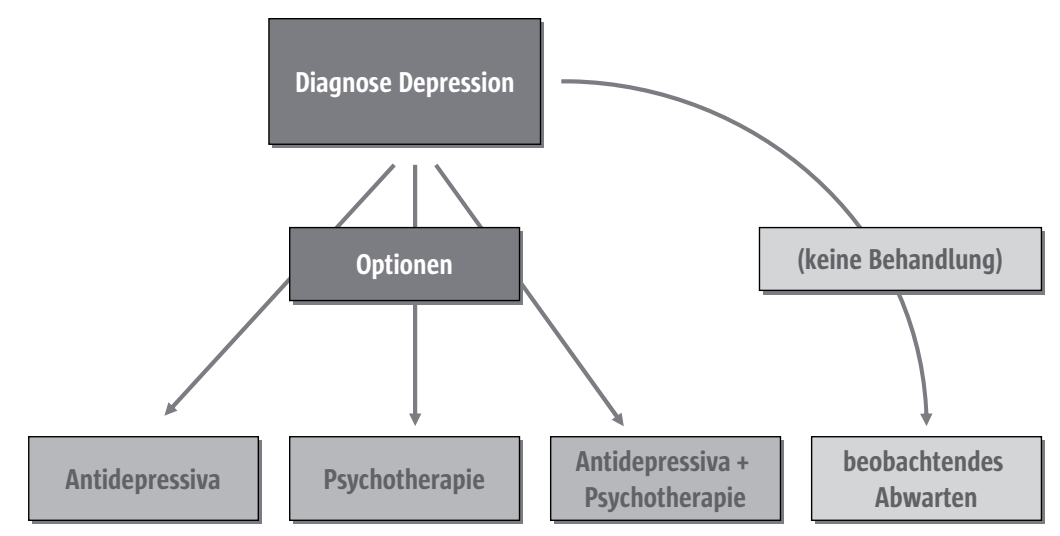

Abb. 15 Entscheidungstafel zu den Behandlungsalternativen in der ambulanten Depressionsbehandlung

Werte reflektieren kann. Patienten bewerten beispielsweise Therapieansätze in der Depressionsbehandlung nicht in erster Linie nach dem Verträglichkeitsprofil, sondern danach, wie schnell und wie wahrscheinlich die erwünschte Wirkung eintreten sollte, welchen Aufwand der Patient zur Realisierung aufbringen muss, welche Ziele mit der Therapie erreicht werden sollen oder welche Wartezeiten bis zum Beginn einer eventuellen Psychotherapie aufgewendet werden müssen (s. Abb. 16). Diese Übersetzungsleistung der Therapiemodalitäten auf die Lebensrealitäten und die Bewertungskriterien aus Patientensicht ist ein wesentlicher Baustein des Abwägens von Optionen im

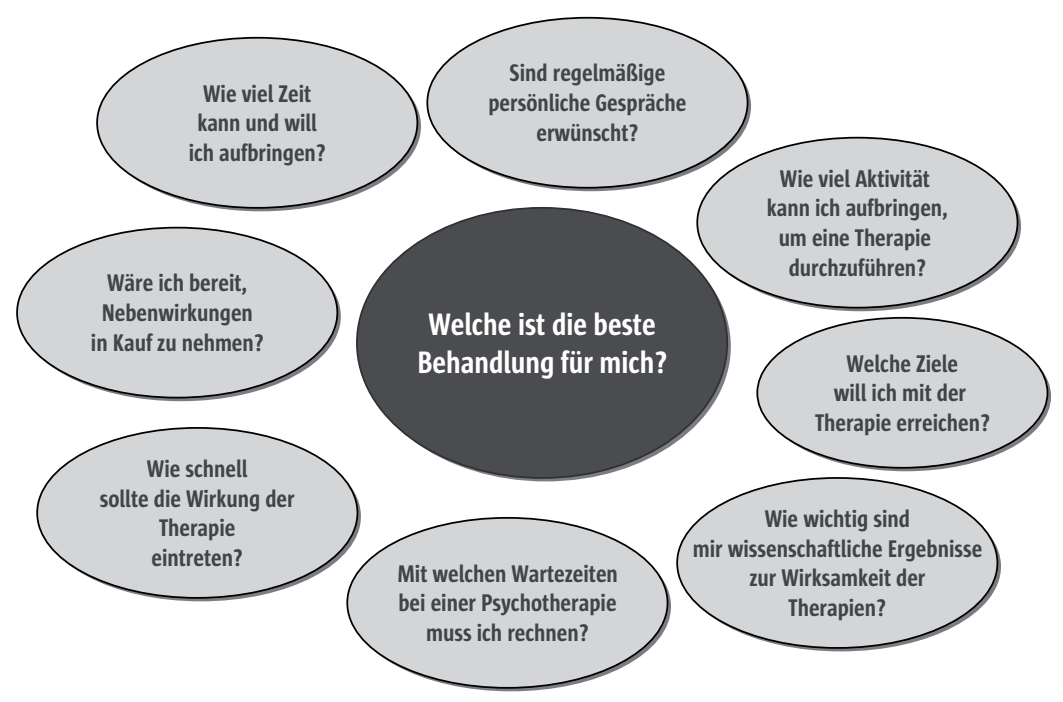

Abb. 16 Patientenfragen zu den Behandlungsmöglichkeiten 
7 Patientenbeteiligung bei medizinischen Entscheidungen als Qualitätsmerkmal der Versorgung am Beispiel der ambulanten Depressionsbehandlung

Arzt-Patienten-Gespräch und damit ein Kernelement im Prozess der Partizipativen Entscheidungsfindung.

Auch bei der Erläuterung der Modalitäten der zur Verfügung stehenden Behandlungen werden sinnvollerweise zur Verdeutlichung graphische oder tabellarische Darstellungen eingesetzt (s. Tab. 15). Die Behandlungsmöglichkeiten sollten kurz und verständlich erläutert werden. Vorteile für die Lebenswelt des Patienten sind in der hier gewählten beispielhaften Darstellung mit

Tab. 15 Vor- und Nachteile der Möglichkeiten zur ambulanten Depressionsbehandlung aus Sicht des Patienten

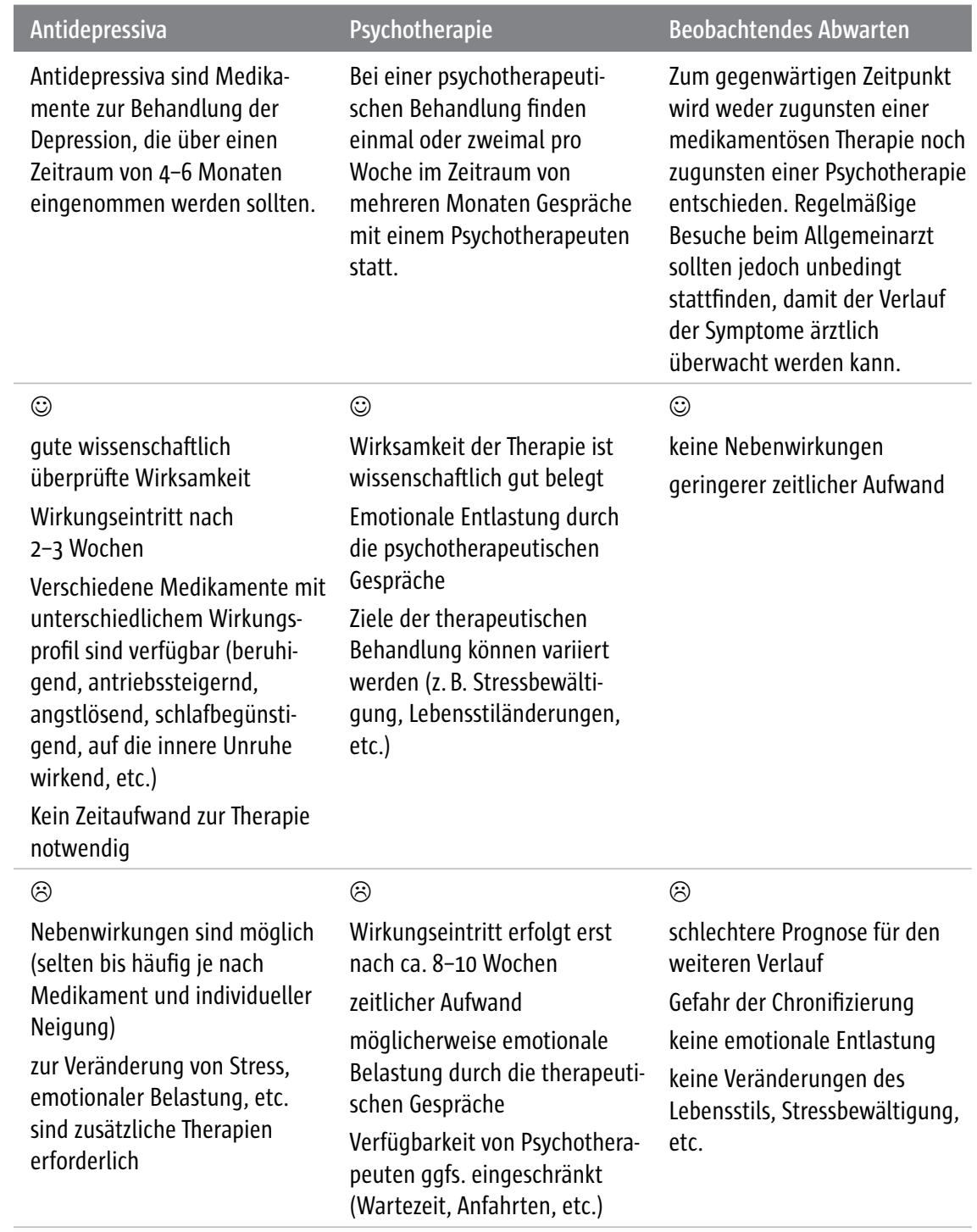


Smileys, d. h. lächelnden Gesichtern, gekennzeichnet, Nachteile mit traurigen Gesichtern. Weitere Informationen sind in den Patienteninformationsmaterialien enthalten und können im Gespräch erörtert werden. Die Option „Beobachtendes Abwarten“ ist im Ansatz der Partizipativen Entscheidungsfindung eine stets zu berücksichtigende Wahlmöglichkeit, über deren Vorund Nachteile ebenfalls wertfrei informiert wird.

\subsection{Welche Erfahrungen machen die Ärzte im Versorgungsalltag mit diesem Ansatz?}

Mittlerweile liegen einige Erfahrungen mit diesem Ansatz vor. Ärzte berichten nach einer Fortbildung und der nachfolgenden Umsetzung in ihrer Praxis, dass das Arzt-Patienten-Gespräch mehr Substanz gewinnt, dass man viel besser mit den Patienten zusammen arbeiten kann und eher eine Atmosphäre entsteht, dass Arzt und Patient ein Team sind und an einem gemeinsamen Strang ziehen. Durch den Einsatz der Entscheidungstafeln wird klarer, um was es geht. Ärzte berichten auch, dass man schneller zu Entscheidungen kommt und die Patienten nicht immer wieder kommen, um das oder jenes noch mal zu bereden. Die getroffenen Entscheidungen sind beständiger. Eine häufige Rückmeldung ist auch, dass die Patienten das viel positiver auf nehmen, als die Ärzte das vor Beginn gedacht haben: „Manchmal ist es anfangs etwas schwer, aber dann machen sie richtig mit - und das ist dann richtig gut."

Bei der Umsetzung in die Praxisroutine ist es erforderlich, eine andere Haltung gegenüber dem Patienten und ein anderes Selbstverständnis in der eigenen Rolle einzunehmen. In den Rollenerwartungen des Arztes an das eigene Verhalten und in den Vermutungen des Arztes zu dem, was er glaubt, was der Patient von ihm erwarten wird, sind häufig solche Haltungen zu finden, die dem Arzt die alleinige Verantwortungsübernahme zuschreiben. Dies scheinen nahezu ritualisierte Verhaltenszuschreibungen zu sein, die mit entsprechender Motivation und Disziplin im ärztlichen Gesprächsangebot verändert werden können. Ärzte mit einer gewissen Erfahrung in diesem Ansatz teilen mit, dass Patienten auf das Angebot der gemeinsamen Entscheidungsfindung zwar zunächst überrascht reagieren, diese Möglichkeit der Beteiligung aber dann in den meisten Fällen gerne annehmen .

Gelegentlich ist zu hören, dass Ärzte befürchten, dass die Patienten dieses Angebot der Ärzte als eine unzureichender Kompetenz des Arztes interpretieren könnten oder als Verweigerung der Annahme seiner professionellen Rolle. Der Eindruck, der Arzt wisse bei dem Problem auch nicht so genau, was eigentlich die beste Behandlung sei, entsteht aber nur dann, wenn der Arzt über die verschiedenen Vor- und Nachteile nicht gut informiert ist. Unter der Voraussetzung der hierfür erforderlichen kommunikativen Kompetenz und des hierfür erforderlichen Wissens des Arztes erleben die Patienten diese Haltung je- 
7 Patientenbeteiligung bei medizinischen Entscheidungen als Qualitätsmerkmal der Versorgung am Beispiel der ambulanten Depressionsbehandlung

doch nicht als eine Einschränkung, sondern eher als Ausweitung der ärztlichen Kompetenz.

Die Hürden zur Implementierung dieses Ansatzes liegen nach wie vor in der nur unzureichenden Anzahl qualitätsgeprüfter Patienteninformationsmaterialien und Entscheidungshilfen und in der Tatsache, dass bisher nur wenige spezifische Trainingsansätze verfügbar sind. Diesbezüglich müssen zunehmende Anstrengungen in der medizinischen Aus-, Fort- und Weiterbildung gefordert werden. Sollten diese allerdings vorliegen und sollten die Patienten den zunehmend in der Forschung auch dokumentierten Wunsch nach Information und Beteiligung in das Arzt-Patienten-Gespräch einbringen, dann erweist sich der Ansatz der Partizipativen Entscheidungsfindung als eine wirksame Methode zur Förderung von Patientenzufriedenheit, Behandlungsmotivation, Behandlungserfolg und zur Stärkung der Arzt-Patienten-Beziehung.

\section{Literatur}

Braddock CH, Edwards KA, Hasenberg NM: Informed decision making in outpatient practice. JAMA 1999; 282: 2313-2320

Bundesministerium für Gesundheit und Soziale Sicherung, Bundesministerium der Justiz 2005: http://www. bmj.bund.de/files/-/1338/Patientenrechte_Aug05.pdf

Charles C, Gafni A, Whelan T: Shared decision making in the medical encounter: what does it mean? (Or it takes at least two to tango). Soc Sci Med 1997; 44: 681-692

Elwyn $\mathrm{G}$ et al. : Developing a quality criteria framework for patient decision aids: online international Delphi consensus process. BM) 2006; 333: 417

Frosch DL, Kaplan RM: Shared decision making in clinical medicine: past research and future directions. Am I Prev Med 1999; 17: 285-294

Härter M, Loh A, Spies C (Hrsg.): Gemeinsam entscheiden - erfolgreich behandeln - Neue Wege für Ärzte und Patienten im Gesundheitswesen. Köln: Deutscher Ärzteverlag 2005

Härter M, Schneider F, Gaebel W et al.: Versorgungsleitlinien für depressive Störungen in der ambulanten Praxis. Supplement IV. Z Arztl Fortbild Qualitatssich 2003; 97: 1-92

Leitliniengruppe Hessen: Homepage der hausärzlichen Leitliniengruppe: http://www.leitlinien.de/leitlinienanbieter/deutsch/pdf/hessengespraech

Loh A, Kremer N, Giersdorf N, Jahn H, Hänselmann S, Bermejo I, Härter M (2004): Informations- und Partizipationsinteressen depressiver Patienten bei der medizinischen Entscheidungsfindung in der hausärztlichen Versorgung. Zeitschrift für ärztliche Fortbildung und Qualität im Gesundheitswesen; 98: 101-107

Loh $A$ et al.: Entwicklung und Evaluation eines Fortbildungsprogramms zur partizipativen Entscheidungsfindung für die hausärztliche Versorgung depressiver Erkrankungen. Bundesgesundheitsblatt - Gesundheitsforschung - Gesundheitsschutz 2004; 47: 977-984

Loh A, Simon D, Bermejo I, Härter M.: Miteinander statt nebeneinander - Der Patient als Partner in der Depressionsbehandlung. In: Härter M, Loh A, Spies C (Hrsg.): Gemeinsam entscheiden erfolgreich behandeln Neue Wege für Ärzte und Patienten im Gesundheitswesen. Köln: Deutscher Ärzteverlag 2005; 165-174

Loh A et al.: Patientenbeteiligung bei medizinischen Entscheidungen: Effekte der Partizipativen Entscheidungsfindung aus systematischen Reviews. Dtsch Arztebl 2007; 104: A-1483

Loh A et al.: Patient and citizen participation in German health care - current state and future perspectives. $Z$ ärztl Fortbild Qual Gesundh wes 2007; 101: 229-235

Loh A, Härter M: Shared decision-making in diverse healthcare systems - translating research and health policy into practice. Z ärztl Fortbild Qual Gesundh wes 2007; 101: 203-204 


\section{Praxisbeispiele}

0'Connor A et al.: Decision aids for people facing health treatment or screening decisions. Cochrane Databse Syst Rev 2002; (3): CD001431.

Scheibler F, Janssen C, Pfaff H: Shared Decision Making: Ein Überblicksartikel über die internationale Forschungsliteratur. Soz Praventivmed 2003; 48: 11-23. 